\title{
TRABAJO COLABORATIVO ENTRE PROFESORES DE EDUCACIÓN DIFERENCIAL Y EDUCACIÓN BÁSICA EN UNA ESCUELA MUNICIPAL*
}

\author{
COLLABORATIVE WORK BETWEEN SPECIAL EDUCATION AND \\ PRIMARY SCHOOL TEACHERS IN A STATE-RUN SCHOOL
}

\section{Fernanda Agüero Andrade**, Nayareth Barriga Leal ${ }^{* * * *}$, Camila Elgueta Riveros****, Daniela Quezada Sáez ${ }^{* * * * *}$, Carmen Paz Tapia Gutiérrez $Z^{* * * * * * *}$}

\section{Resumen}

El presente artículo corresponde a una investigación cualitativa realizada en una escuela municipal ubicada en la ciudad de Temuco, Chile. Participan profesores de educación especial y educación básica, quienes realizaron un trabajo colaborativo. El objetivo de esta investigación es describir el trabajo colaborativo realizado en conjunto e identificar facilitadores y obstaculizadores presentes en el proceso. Para llevar a cabo la investigación se utilizó un diseño y paradigma hermenéutico, por lo que se recolectaron los datos mediante la observación no participante y entrevista semiestructurada.

* Proyecto de investigación para obtener grado de Licenciadas en Educación.

** Profesora de Educación Diferencial con especialización en NEET y Deficiencia Mental, Universidad Católica de Temuco, email: faguero2012@alu.uct.cl

*** Profesora de Educación Diferencial con especialización en NEET y Deficiencia Mental, Universidad Católica de Temuco, email: nbarriga2015@alu.uct.cl

**** Profesora de Educación Diferencial con especialización en NEET y Deficiencia Mental, Universidad Católica deTemuco, email: celgueta2015@alu.uct.cl

***** Profesora de Educación Diferencial con especialización en NEET y Deficiencia Mental, Universidad Católica de Temuco, email: dquezada2015@alu.uct.cl

******* Magíster en Pedagogía y Gestión Universitaria Facultad de Educación. Universidad Católica de Temuco, email: ctapia@uct.cl 
Finalmente, se utilizó el análisis de contenido y de tipo deductivo, lo que permitió obtener como resultados la existencia de un trabajo en un mismo horario y espacio, con acciones en común. Además, se identificaron facilitadores como la relación entre pares; y obstaculizadores como la falta de orientación para realizar el trabajo, los que, en base a la teoría, son un factor común en Chile.

Palabras clave: Educación, docente, docente de escuela primaria, profesor especializado, participación del profesor, planificación de la educación.

\begin{abstract}
This article reports a qualitative study conducted in a public school located in Temuco, Chile. The participants are special education and primary school teachers, who develop collaborative work. The objective is to describe the collaborative work they do together and to identify advantages and disadvantages during the process. A hermeneutical design and paradigm is used, so the data were collected through a non-participant observation and a semi-structured interview. Finally, deductive content analysis was used, which allowed to obtain as results the existence of a job done at the same time and space, with common actions. In addition, advantages like peer relationship, and obstacles such as the lack of orientation to carry out the work were identified, which based on the theory represents a common factor in Chile.
\end{abstract}

Keywords: Education, teachers, primary school teachers, special education teachers, teacher participation, educational planning.

\title{
1. Introducción
}

AS INSTITUCIONES EDUCATIVAS se caracterizan por su diversidad, por lo que en los últimos años ha surgido la necesidad de dar respuestas que beneficien a todos los estudiantes mediante distintas normativas a nivel nacional. No obstante, hay contradicciones entre lo teórico y lo práctico como se observa en el trabajo entre el profesorado de las instituciones, situación que repercute en el aprendizaje de los estudiantes.

El trabajo colaborativo, según Rodríguez (2014), es una estrategia 
utilizada para el aprendizaje de los estudiantes. Para desarrollar este trabajo se requiere conocer las características de los estudiantes, lo que se ve evidenciado y potenciado en el proceso de enseñanzaaprendizaje que realizan los profesores para conocer a los estudiantes y responder a sus necesidades, en conjunto con los profesionales de educación diferencial.

En el decreto 83 del año 2015 del Ministerio de Educación de Chile (MINEDUC), se establecen nuevas orientaciones y criterios sobre la diversificación de la enseñanza, donde se plantea que es tarea de todos los docentes. En consecuencia, se otorgan horarios de planificación en conjunto, en que deben participar todos los profesores, con el objetivo de diseñar estrategias de enseñanza aprendizaje y evaluación que consideren a todos los estudiantes. Sin embargo, existen investigaciones en donde se evidencia que dicho trabajo no se lleva a cabo de manera eficiente. Por esta razón, la Escuela Municipal de Temuco toma la decisión e iniciativa de establecer un mismo horario de trabajo colaborativo para todos los profesores de la institución y en un mismo espacio, definido en la sala de profesores.

Ante esta nueva realidad, surge la necesidad de comprender y analizar la dinámica de trabajo en la escuela, para describir cómo la llevan a cabo e identificar los facilitadores y obstaculizadores de esta nueva modalidad. La pregunta de investigación que se responderá en la presente investigación es ¿Cómo se trabaja colaborativamente entre profesores de educación diferencial y educación básica en la escuela municipal?

El objetivo general de la investigación es: Comprender el trabajo colaborativo entre profesores de educación regular y educación diferencial, entendiendo la dinámica de ambos profesionales. Los Objetivos específicos son los siguientes:

1. Describir el trabajo colaborativo entre profesor de aula y diferencial para la comprensión de la realidad en la que se sitúan.

2. Identificar elementos facilitadores y obstaculizadores del trabajo colaborativo entre profesor de aula y profesor de educación diferencial para la comprensión de la modalidad de trabajo. 


\section{Educación especial y necesidades educativas especiales}

En Chile, se define la educación especial como una modalidad del sistema educativo, que se imparte tanto en las escuelas de educación regular, como en escuelas especiales. La finalidad es asegurar la igualdad de oportunidades y de participación de todas las personas, ya sean niños, jóvenes o adultos que presenten necesidades educativas especiales (MINEDUC, 2005).

En los años 70 y especialmente en los años 80, es el momento en que empieza a cuestionarse este modelo de organización de la educación especial. A partir de aquí se produce un fuerte movimiento integrador de los alumnos con necesidades educativas especiales en los centros ordinarios (Font, 2009, p. 113).

Con el informe Warnock de 1978, surge el concepto de necesidades educativas especiales y se logra un "cambio de perspectiva que amplía la concepción de la educación especial, dejando atrás las visiones que consideran a la especial y la general como realidades separadas" (MINEDUC, 2005, p. 10). Ante esto, el estado de Chile se adscribe, creando políticas y marcos legales que favorezcan el derecho a la educación.

A partir de ello, los principales avances educacionales de educación especial en Chile (MINEDUC, 2005, p. 17) son:

- Incremento de la subvención de la educación especial en un $330 \%$.

- Normativas que permitieron la integración escolar de los alumnos con discapacidad al sistema de educación regular (Decretos Supremos de Educación No 490/90 y No 1/98, que reglamenta el Título IV, Capítulo II de la Ley $\mathrm{N}^{\circ} 19.284$ ).

- Aumento de cobertura de la integración de niños y jóvenes con necesidades educativas especiales al sistema de educación regular. - Más de 20.000 profesores de educación especial y regular, entre los años 1998 y 2005, participaron en actividades de información y perfeccionamiento con el propósito de promover la integración escolar.

- A fines del año 2003, el Ministerio de Educación se comprometió a elaborar una nueva política para la educación especial.

Posteriormente, se observan los siguientes cambios significativos: 
- En el año 2009, la Ley General de Educación establece la necesidad de considerar las necesidades educativas especiales.

- En el mismo año, en el decreto 170, el profesor de educación diferencial fue integrado al aula regular.

- En el año 2015, se promulga el Decreto 83, donde se establecen horarios de trabajo colaborativo.

En relación a la evolución de la consideración social y educativa de las personas con discapacidad y Necesidades Educativas Especiales (en adelante NEE), Godoy, Meza y Salazar (2004) establecen que la población con discapacidad es la que ha sufrido de mayor discriminación en el sistema social, educacional y laboral.

En la década del 80 y 90, se abandonó el enfoque centrado en el déficit para situarse en un marco propiamente educativo, el que da respuesta a un enfoque más holístico, centrado en los procesos de enseñanza-aprendizaje de los estudiantes, lo que permitió avanzar, según la Organización de las Naciones Unidas para la Educación, la Ciencia y la Cultura (desde ahora UNESCO) a una inclusión educativa, ya que señala que:

La inclusión es un movimiento orientado a transformar los sistemas educativos para responder a la diversidad del alumnado. Es fundamental para hacer efectivo el derecho a la educación con igualdad de oportunidades y está relacionada con el acceso, la permanencia, la participación y los logros de todos los estudiantes, con especial énfasis en aquellos que por diferentes razones, están excluidos o en riesgo de ser marginados (Juárez, Comboni y Garnique, 2010, p. 49).

\section{Aprendizaje y trabajo colaborativo}

En las instituciones escolares, una de las estrategias utilizadas para dar respuesta a la diversidad del estudiantado es el trabajo colaborativo, que Rodríguez (2014) define como "una de las principales estrategias organizacionales y curriculares utilizadas para el aprendizaje desde un enfoque inclusivo" (p. 304). Poder desarrollarlo implica tener un conocimiento de las habilidades propias como docente y además de los estudiantes, es por ello que "el aprendizaje es una actividad crucial en cualquier cultura humana. 
La existencia misma de una cultura depende de la capacidad de los nuevos miembros para aprender habilidades, normas de comportamiento, hechos y creencias"(Anderson, 2002, p. 78). Esto se ve potenciado mayormente en la escuela, en donde los profesores están a cargo del proceso de enseñanza-aprendizaje.

Ante esto, el decreto de la Diversificación de la Enseñanza (Ministerio de Educación, 2015) estableció la obligatoriedad de otorgar horas a los profesores para realizar trabajo colaborativo en los establecimientos educacionales, con Programas de Integración Escolar que den una respuesta educativa ajustada a los estudiantes y que también potencien las habilidades docentes (ver Cotrina, García y Caparrós, 2017).

\section{Facilitadores y obstaculizadores para implementar el trabajo co- laborativo en la actualidad}

En Chile, como se mencionó anteriormente, existieron cambios a nivel político que contribuyeron a la educación especial y general, lo que a su vez implicó cambios en los marcos referenciales de los profesores. Es por ello que el Marco para la Buena Enseñanza menciona en el dominio D: Responsabilidades Profesionales, que el profesor debe comprometerse a evaluar los procesos de aprendizaje de sus estudiantes para comprenderlos, levantando fortalezas y necesidades y entregándoles apoyos pertinentes. También se requiere que forme parte constructiva del entorno donde trabaja, es decir, que comparta, aprenda de sus colegas y con ellos, trabajar en equipo (MINEDUC, 2008).

Una de las formas de trabajo en equipo es el trabajo colaborativo que se define como "una de las principales estrategias organizacionales y curriculares utilizadas para el aprendizaje desde un enfoque inclusivo" (Rodríguez, 2014, p. 304). Es importante señalar que, si bien el trabajo colaborativo se debe desarrollar desde distintas disciplinas, la presente investigación se focaliza en el trabajo colaborativo entre el profesor de aula regular y profesor de educación diferencial.

Desde está lógica, el trabajo colaborativo entre los profesores "considera nuevas alternativas y re-elabora ideas tanto para comunicarlas como para fundamentar lo que se proponen los demás” (Rotstein, 
Scassa, Sáinz y Simesen, 2006, p. 39). Las autoras además señalan que, el trabajo colaborativo permite que todos los miembros aprecien el esfuerzo y aporte del otro, compartan una meta, responsabilidades, organizaciones e implicancia personal, aporten estrategias comunicaciones y operativas para promover el aprendizaje. Asimismo, por medio de la interacción recíproca, comparten, se retroalimentan, evalúan y debaten ideas que incrementan la motivación por el aprendizaje y el vínculo solidario, por medio de la confrontación de experiencias interpersonales y desarrollo de colaboración entre pares (Rotstein et al., 2006).

Con el trabajo colaborativo, los profesores se ven beneficiados en: "el desarrollo de un sentido de comunidad, una mayor motivación, crecimiento profesional y sentido de satisfacción con el trabajo" (ver Rodríguez, 2014, p. 222), lográndose, de esta forma, un trabajo en comunidad entre los profesores, que contribuye a aumentar su motivación para trabajar en conjunto y sentirse satisfechos de lo realizado.

Maldonado (2008) también señala que otro de los beneficios del trabajo colaborativo es que contribuye a aumentar la seguridad en sí mismo, pero al mismo tiempo estimula el desarrollo del pensamiento crítico, fortalece el valor de la solidaridad y respeto recíproco, a la vez que reduce los sentimientos de aislamiento. De igual forma, Jiménez y Jiménez (2004) reconocen la colaboración como una forma de enriquecimiento mutuo y como estrategia de desarrollo de habilidades.

López (2011), en una de sus investigaciones, resalta los facilitadores del trabajo colaborativo, donde se encuentran en primer lugar las relaciones personales afectuosas y cordiales entre los profesores. En segundo lugar es importante que no haya muchos miembros dentro de este trabajo, ya que "es más fácil mantener la interacción cuando la escuela es pequeña debido a que no hay muchos miembros implicados y esto agiliza la comunicación y coordinación de actividades” (p. 94). En tercer lugar se encuentra "la existencia de competencias comunes que se busca promover y que demanda el desarrollo de un trabajo conjunto entre docentes"(p. 95), compartiendo un mismo objetivo y meta para contribuir a un trabajo más fluido y fácil de articular. Mientras que en cuarto lugar es relevante la actitud positiva de los docentes hacia el trabajo colaborativo, destacándose la importancia 
de trabajar en conjunto para llevar a cabo el trabajo de forma efectiva y satisfactoria.

Por otro lado, Rodríguez (2012) plantea que "en Chile se han hallado indicios de que el trabajo colaborativo se ve dificultado por un problema de organización escolar" (p. 226), ya que, según los profesores, los tiempos designados para la realización del trabajo colaborativo muchas veces no coinciden con las de los otros profesores, transformándose en un obstaculizador.

Además, Berrios, Calderón y Pizolty (2016), señalan que las problemáticas han sido estudiadas a nivel nacional e internacional y estas "coinciden con identificar que el rol de liderazgo en esta relación generalmente recae en el profesor regular por sobre el diferencial" (p. 357). Además, mencionan que el profesor de educación diferencial es visto sólo como un apoyo y no como un par, lo que se puede relacionar directamente con el desconocimiento del rol del educador diferencial y provoca que la implementación de estrategias no se desarrolle de forma oportuna.

Por otro lado, Sanhueza, Granada y Bravo (2013), en su investigación identificaron que los principales problemas que tienen los profesores de educación básica y de educación diferencial son la falta de recursos y tiempos para abordar las tareas educativas de planificar y colaborar con otros.

Navarro, Arriagada, Osse y Burgos (2016) también mencionan que la "planificación de las adaptaciones curriculares es deteriorada por la inexistencia de tiempo formal, es decir, tiempo dentro de la carga horaria destinada al trabajo con estudiantes con NEET o para planificar y atender a dichas necesidades" (p. 13).

En Chile, algunos profesores de aula regular y profesores de educación diferencial no están trabajando de manera colaborativa dentro del aula regular, lo que repercute en el aprendizaje de los estudiantes. En este contexto, Rodríguez y Ossa (2014) evidenciaron, en su investigación, que los profesores no poseen horas suficientes para realizar el trabajo colaborativo, por lo que deben "utilizar tiempo informal por causa de problemas de contratación y coordinación de las horas entre los docentes" (p. 308).

Según los autores, las dificultades y obstaculizadores en el desarrollo 
del trabajo colaborativo son la falta de tiempo y articulación entre los profesores para realizar dicho trabajo, falta de recursos que faciliten su desarrollo y el liderazgo en las clases es ejercido por el profesor de aula regular, mientras que el profesor diferencial ejerce un papel más pasivo.

\section{Métodos y materiales}

En esta investigación se utilizará el enfoque cualitativo, el cual:

Facilita una recogida de datos empíricos que ofrecen descripciones complejas de acontecimientos, interacciones, comportamientos y pensamientos, que conducen a la interpretación de los datos. En este sentido, el diseño cualitativo está unido a la teoría, en cuanto que se hace necesario una teoría que explique, que informe e integre los datos para su interpretación (Quecedo y Castaño, 2002, p. 12).

Sobre la base de esta información, en el proceso de la actual investigación, se recogerán datos de las percepciones y experiencias de los profesores de aula regular y profesores de educación diferencial sobre el trabajo colaborativo entre ellos, a través de distintos instrumentos de recogida de información, en donde se garantiza una relación constante y dinámica entre las preguntas de investigación, la recaudación de datos y la posterior interpretación. Todo ello para responder al objetivo general y a los objetivos específicos, orientados a comprender, identificar, describir y analizar las distintas situaciones vivenciadas.

\subsection{Contexto y participantes}

La investigación se realizó en una escuela municipal de Chile, cuya visión institucional es acoger las distintas necesidades y realidades convergentes del entorno en el que se encuentra, entregando a todas y todos los estudiantes un desarrollo integral, crecimiento individual, compromiso ético, psicosocial y cultural. Esta escuela posee una enseñanza que abarca la educación parvularia y la educación básica, de donde se ha seleccionado una muestra de tipo por conveniencia, dado que: "El investigador decide qué individuos de la población pasan a 
formar parte de la muestra en función de la disponibilidad de los mismos" (Canal, 2006, p. 126).

A partir de ello, se contemplaron los siguientes criterios de inclusión:

- Profesores de educación básica que trabajen en conjunto con profesores de educación diferencial, para la realización de planificaciones y clases.

- Profesores de educación diferencial para que trabajen en conjunto con profesores de educación básica, para la realización de planificaciones y clases.

Para la descripción de los participantes, se registraron los datos de: edad, sexo y tiempo de antigüedad en la institución.

Los criterios de inclusión cuentan con una codificación, que es comprendida como un código asignado a los participantes, es por ello que:

- Las profesoras de educación básica: E_PB_1 y E_PB_2.

- Las profesoras de educación diferencial: E_ED_1 y E_ED_2.

El total de participantes de la investigación dependió de los instrumentos aplicados. Así, en relación a la entrevista semiestructurada, participaron 2 profesoras de educación básica y 2 de educación diferencial. Mientras que, en la observación, se contempló un total de 15 profesores, de los cuales 12 eran de educación básica y 3 de educación diferencial.

\subsection{Técnicas}

Uno de los instrumentos a utilizar, para la recogida de información, fue la observación de la realización del trabajo colaborativo entre los profesores. Según Merchán y Ramírez (2015), este instrumento permite a los investigadores identificar, describir y entender el funcionamiento de las acciones realizadas por los observados.

También, se elaboró una entrevista de tipo semiestructurada, ya que "es característico de esta entrevista que se traigan a la situación de entrevista, preguntas más o menos abiertas en forma de guía de entrevista. Se espera que el entrevistado responda a ellas libremente" (Flick, 2004, pp. 106-107).

Con ambos instrumentos, se pretendió describir el actuar de los profesores de educación diferencial y de educación regular durante las instancias de trabajo colaborativo, con el propósito de identificar las 
fortalezas y debilidades. Mientras que las entrevistas se aplicaron a profesores de educación regular y de educación diferencial, lo que permitió recoger puntos de vista de los entrevistados, registrando de esta manera las fortalezas y dificultades que ellos visualizan en estas instancias de trabajo colaborativo.

\subsection{Validación}

Los instrumentos de evaluación, antes de ser implementados, se enviaron a una profesora de la carrera de pedagogía en educación diferencial, experta en trabajo colaborativo, presentándole la investigación, instrumentos a validar y carta de certificación de validez.

Posteriormente, se recibió retroalimentación de parte de la docente y se realizaron distintas modificaciones a los instrumentos. Finalmente, tras 2 modificaciones a los instrumentos, la experta otorga su firma de validación de los instrumentos, señalando que "los instrumentos son coherentes con los objetivos de la investigación, así que se encuentran en óptimas condiciones para ser aplicados".

\subsection{Procedimientos}

Desde los procedimientos, se informó al establecimiento educativo, posteriormente se entregó una carta a la directora del establecimiento y al encargado de Unidad Técnica Pedagógica. Luego se expuso al cuerpo docente la temática, seleccionando los participantes que serían parte de la muestra, entregándoles el consentimiento informado, donde dejan constancia que aceptan trabajar en la investigación.

Se comenzó a implementar la pauta de observación del trabajo colaborativo entre los profesionales seleccionados, para posteriormente llevar a cabo las entrevistas semiestructuradas a los participantes. La información obtenida, por medio de la observación y las entrevistas, fue consensuada en una primera instancia para, posteriormente, ser planteada en una matriz de vaciado de información, permitiendo analizar y contrastar los diferentes puntos de vista de los profesores. 


\subsection{Análisis de los datos}

\section{a) Tipo de análisis}

En la investigación educativa se hizo uso de un análisis de contenido, ya que "se basa en la lectura (textual o visual) como instrumento de recogida de información, lectura que a diferencia de la lectura común, debe realizarse siguiendo el método científico, es decir, debe ser sistemática, objetiva, replicable, y válida” (Abela, 2002, p. 2). La recogida de información fue desarrollada por dos instrumentos de evaluación: la entrevista semiestructurada y la observación no participante. Posteriormente, se realizó un análisis de contenido de corte de análisis de tipo deductivo para reducir los datos recolectados en el proceso de la aplicación de los instrumentos, en donde, el primer objetivo específico da cuenta de "describir el trabajo colaborativo entre profesor de aula regular y diferencial para la comprensión la realidad en la que se sitúan”.

El análisis deductivo "permite establecer un vínculo de unión entre teoría y observación y permite deducir a partir de la teoría los fenómenos de objeto de observación"(Dávila, 2006, p. 181). Por lo tanto, se utilizarán las categorías que se establecieron previamente, permitiendo llegar a una conclusión de los datos que se recogieron. Además, es necesario realizar una vinculación de unión entre la teoría y la observación, debido a que se plantea en los objetivos: generar un análisis que permita identificar elementos facilitadores y obstaculizadores del trabajo colaborativo, siendo necesario recoger información a partir de la observación no participativa y de la entrevista semiestructurada.

\section{b) Tipo de triangulación}

Se utilizó una triangulación metodológica, que consiste en "la combinación de varios métodos de recogida y análisis de datos para acercarse a la realidad investigada" (Ardoy, Pasadas del Amo y Ruiz, 2004, p. 2) permitiendo identificar si los datos son temporales, es decir, datos recogidos en distintas fechas para comprobar si los resultados son constantes o si es espacial, que quiere decir que si se comprueban coincidencias. De esta forma, incrementamos la calidad y la validez de datos, puesto que se cuenta con distintas perspectivas de un mismo estudio. 


\subsection{Procedimiento de Análisis de datos}

Para el análisis de datos, en primer lugar, se realizó la transcripción de las entrevistas y su posterior pisteo para generar unidades de análisis; con las que se establecieron categorías y subcategorías. Para la entrevista semiestructurada, respondiendo al objetivo Describir el trabajo colaborativo entre los profesores, se levantaron las categorías de Nueva Organización del Trabajo Colaborativo y Antigua Organización, desde la primera categoría se desglosaron las siguientes subcategorías: Horario, Participantes, Causa del Cambio, Beneficios, Evaluación del Trabajo Colaborativo y Habilidades Desarrolladas en el Trabajo Colaborativo.

Mientras que en el objetivo No 2, Identificar elementos facilitadores y obstaculizadores del trabajo colaborativo entre los profesionales, se levantaron categorías de Facilitadores y Obstaculizadores del trabajo colaborativo, y para la observación no participante, que responde a ambos objetivos específicos, se levantaron tres categorías: Horario y Espacio Físico, Participantes, Acciones Desarrolladas: se comprende por fase de Inicio, Desarrollo y Cierre.

Luego se procede a vaciar la información en matrices de análisis, las que se distribuyen por objetivo y por instrumento de evaluación, para luego generar una descripción y triangulación de los resultados como se evidencia en los resultados. Ver figura 1.

Figura 1. Resumen del proceso de análisis de datos

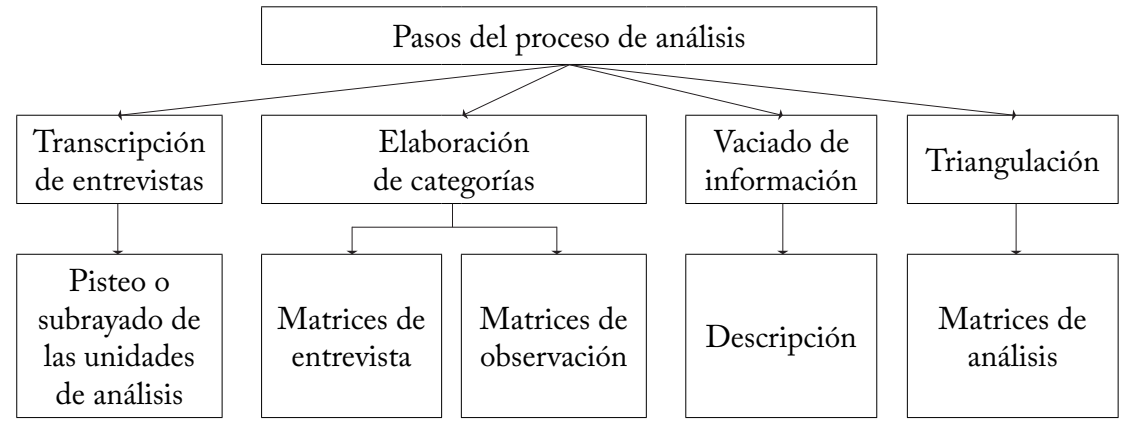

Fuente: Creación propia 


\section{Resultados}

A partir del año 2018, se comenzó a implementar una nueva modalidad de trabajo colaborativo en la Escuela, en donde se señala que la causa del cambio surge al notar que no se generaban instancias para trabajar en conjunto, presentando dificultades para coordinar espacios y horarios, lo que repercutió en la falta de efectividad del trabajo en el aula.

A continuación, se procede a representar la organización del trabajo colaborativo a través de la figura $\mathrm{N}^{\circ} 2$, describiendo las acciones que llevan a cabo el profesor de educación de diferencial y educación básica, para posteriormente destacar el aporte de esta modalidad desde la percepción de los profesores.

Figura 2. Organización del Trabajo Colaborativo

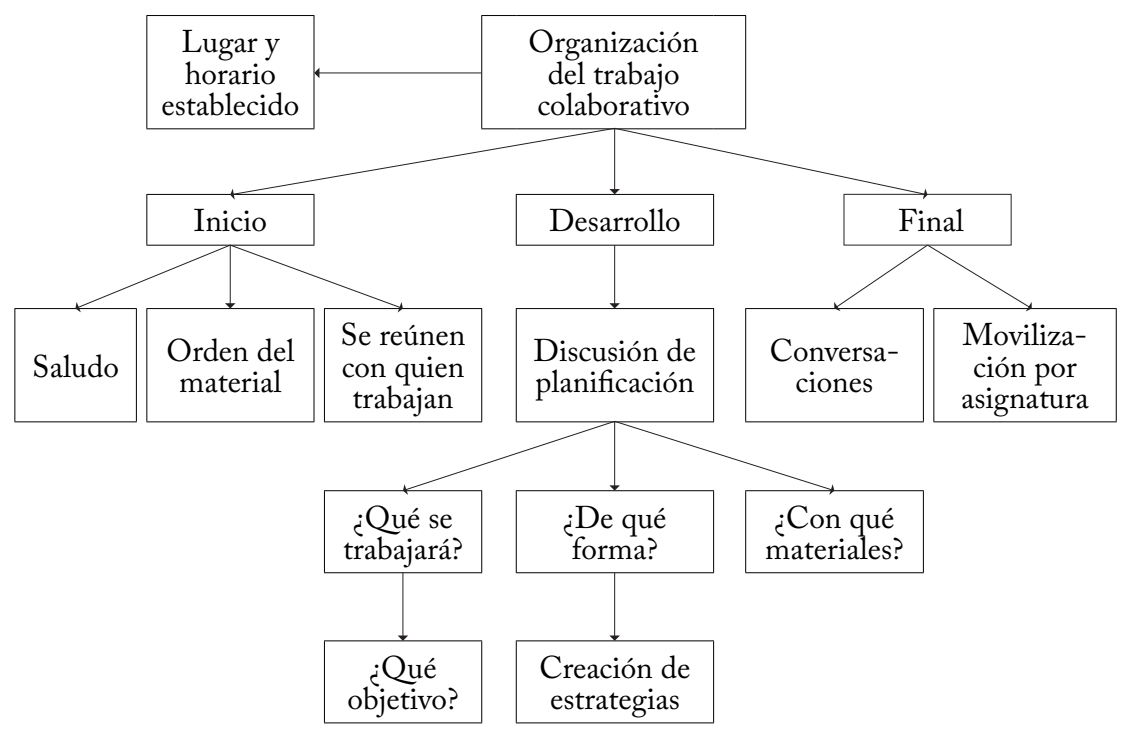

Fuente: Creación propia

Los profesores señalan que cuentan con 3 horas de Trabajo Colaborativo, en donde 2 se realizan el día martes de 15:30 a 17:15 horas, en 
un espacio en común, y una hora de trabajo autónomo que se distribuye según el horario de cada profesor.

En las reuniones de los días martes, se trabaja en la coordinación del proceso de enseñanza-aprendizaje, por medio de la realización de planificaciones, estructurando los objetivos de trabajo, el contenido y la estrategia a utilizar para el desarrollo de la secuencia didáctica.

Desde las observaciones llevadas a cabo, fue posible identificar 3 momentos en donde desarrollan las acciones pedagógicas:

- Inicio: Los profesores llegan a la sala de profesores, se saludan, organizan su material de trabajo, para finalmente reunirse con su par de trabajo.

- Desarrollo: Los profesores preparan la planificación, y se inician discusiones en torno a qué se trabajará, de qué forma, qué materiales serán incorporados en la clase y qué rol tendrá cada uno durante el desarrollo de la clase. En esta fase, surgen también algunas dudas, las que son comentadas a nivel general entre los distintos profesores. Solo en una ocasión, se habló sobra la forma en que evaluarían a los estudiantes.

- Final: Al finalizar, el educador diferencial se dirige hacia el otro profesor de educación básica con quien le corresponde trabajar. Cuando todos terminan de trabajar se inician conversaciones, donde se abordan temáticas complejas de algunos estudiantes, planificaciones a nivel institucional y algunos comentarios de su vida personal.

Estos 3 momentos son similares en todos los trabajos colaborativos que se llevan a cabo en la sala de profesores, sólo variando el orden de las acciones en el desarrollo.

Posteriormente, cada profesor de educación básica tiene un tiempo determinado para trabajar con el educador diferencial, planificando, en conjunto, las actividades a realizar, y una vez finalizado el trabajo el educador diferencial se traslada hacia el otro profesor de educación básica con quien le corresponde trabajar, para iniciar nuevamente el mismo proceso de planificación. En estas instancias, también se evidenció que los profesores interactúan entre ellos al solicitar estrategias y retroalimentaciones entre la totalidad del grupo participante. 


\subsection{Aportes del trabajo colaborativo: Desarrollo del proceso de enseñanza-aprendizaje}

Según las percepciones de ambos profesionales, el trabajo colaborativo que llevan a cabo ha contribuido a mejorar el aprendizaje de los estudiantes porque pueden estructurar la enseñanza con nuevas estrategias, surgidas a partir del trabajo entre pares. En relación a esto, se menciona que "han sido avances significativos en el aprendizaje de los niños, sobre todo por el tema de aplicar nuevas estrategias en el aula." (E_ED_1).Además, otro profesor dice que "la experiencia de los pares ayuda mucho en esto, porque uno se enriquece"(E_PB_1), destacándose un proceso de retroalimentación entre los profesores para una toma de decisiones que contribuyan a los aprendizajes de los estudiantes.

\subsubsection{Fortalecimiento de la relación entre dupla}

Otro de los aportes que destacan los profesores es el desarrollo de una mejor comunicación entre ellos. Esto implica que el trabajo colaborativo ha contribuido a fortalecer sus relaciones, ya que los principales elementos que se han visto beneficiados son "la comunicación que se ha dado, la confianza... el compañerismo" (E_PB_1).

Se evidencia que los profesores de la Escuela Municipal trabajan colaborativamente en un mismo horario y en un espacio en común. Esta modalidad, ha aportado múltiples beneficios a los profesores, tales como: el desarrollo de la enseñanza-aprendizaje y el fortalecimiento de la relación entre las duplas de trabajo.

\subsubsection{Facilitadores y Obstaculizadores del Trabajo Colaborativo}

Para identificar los facilitadores y obstaculizadores del trabajo colaborativo, se desarrolló una entrevista semiestructurada y una observación no participante en las horas de trabajo colaborativo, los resultados se muestran en la Tabla $\mathrm{N}^{\circ} 1$ y serán explicadas posteriormente. 
Tabla N¹. Facilitadores y Obstaculizadores del Trabajo Colaborativo

\begin{tabular}{|c|c|c|}
\hline Criterio & Valoración & Descriptor \\
\hline \multirow[b]{2}{*}{$\begin{array}{l}\text { Condiciones } \\
\text { de organización }\end{array}$} & Facilitadores & $\begin{array}{l}\text { Trabajo en un horario en común. Cuentan } \\
\text { con un espacio para la realización del trabajo } \\
\text { colaborativo. }\end{array}$ \\
\hline & Obstaculizadores & $\begin{array}{l}\text { Requieren de apoyo en otras asignaturas. } \\
\text { No cuentan con planificaciones. Falta de } \\
\text { orientaciones por parte de la institución ante } \\
\text { una nueva estrategia de trabajo. }\end{array}$ \\
\hline \multirow[t]{2}{*}{$\begin{array}{l}\text { Relaciones } \\
\text { entre pares }\end{array}$} & Facilitadores & $\begin{array}{l}\text { Los profesores de educación básica, cuentan } \\
\text { con una comunicación directa con la educa- } \\
\text { dora diferencial. } \\
\text { Fomento de relación entre pares. }\end{array}$ \\
\hline & Obstaculizadores & $\begin{array}{l}\text { Aumento de inasistencia por parte de los } \\
\text { profesores. }\end{array}$ \\
\hline \multirow[b]{2}{*}{$\begin{array}{l}\text { Trabajo } \\
\text { colaborativo }\end{array}$} & Facilitadores & Voluntad ante la colaboración. \\
\hline & Obstaculizadores & $\begin{array}{l}\text { No existe una evaluación formal del trabajo } \\
\text { colaborativo. Escasa claridad de las acciones. }\end{array}$ \\
\hline
\end{tabular}

Fuente: Creación propia

\subsubsection{Facilitadores del Trabajo Colaborativo}

En relación a los facilitadores del trabajo colaborativo, se evidencia el trabajo en un horario y lugar en común, como expresa; "se establece un horario de trabajo colaborativo, entonces estos horarios los cumplimos y vamos viendo el contenido que se va a pasar la próxima clase" ( $E_{-}$ ED_1), donde además se señala "que todos tengamos un horario... en el cual podamos reunirnos” (E_PB_2). Este horario establecido se lleva a cabo los días martes en la sala de profesores, en un horario de 15:30 a 17:15 hrs.

También, los profesores destacan que el Trabajo Colaborativo ha permitido la relación entre pares, señalando que esta metodología ha propiciado: "la relación entre pares, ya que es muy importante, bueno fundamental” (E_ED_1), "nos aportamos entre todos, o sea, intercambiamos experiencias" (E_PB_2). La totalidad de profesores se reúnen en la sala de profesores durante el horario de trabajo colaborativo, 
existiendo un trabajo entre pares, y al presentar dudas, se comenta entre los profesores de las distintas asignaturas. Además, el trabajo colaborativo involucra una comunicación directa como señalan los profesores, identificando que uno de los facilitadores es "tener una comunicación directa con la educadora y con los pares”(E_PB_1). Esto se logra desarrollar al tener todos la misma instancia de trabajar en conjunto.

Y, por último, otro de los facilitadores del trabajo colaborativo, es la existencia de la voluntad de colaboración de los profesores para llevar a cabo un trabajo efectivo que propicie el aprendizaje de los estudiantes, adoptando una actitud positiva ante las dificultades de aprendizaje de los estudiantes, tal como señaló una de las profesoras, quien destaca que en el trabajo llevado a cabo "existe la voluntad, la colaboración" (E_PB_1).

\subsubsection{Obstaculizadores del Trabajo Colaborativo}

En cuanto a los obstaculizadores del trabajo colaborativo evidenciados, se destaca la falta de tiempo en relación a la cantidad de trabajo que deben realizar y el requerimiento de apoyo en otras asignaturas, debido que "debería ser más el tiempo de trabajo colaborativo". Así:

Si piensas que son dos horas para todas las horas de lenguaje y todas las horas de matemáticas, se hace poco y estás focalizando solo en esas dos asignaturas...También necesito de repente ideas en historia, en ciencias que también son emm... digamos, asignaturas duras (E_EB_1).

Esto se ve reflejado en las horas de trabajo, donde focalizan su atención en las asignaturas de lenguaje y matemáticas y no se abordan las demás asignaturas.

Además, el no contar con las planificaciones, "algunos docentes las planificaciones no las tienen, algunas veces están planificando en el momento", "cosa de adecuar la planificación y no ver en el momento qué voy a hacer", "así se podría retroalimentar mucho mejor o ir más avanzadas", "hay algunos que ni siquiera empiezan a trabajar" (E_ED_2). En el desarrollo del trabajo colaborativo, se identifica que los profesores de educación diferencial y educación básica realizan la planificación en el momento o se distribuyen las partes de las planificaciones 
para avanzar de forma autónoma.

Desde otra perspectiva, se identifica una falta de orientaciones por parte de la institución ante una nueva estrategia de trabajo. Como fue comentado en el objetivo $\mathrm{N}^{\circ} 1$, la escuela se encuentra implementando una nueva modalidad de trabajo colaborativo, ante esto se señala que "a principio de año nadie sabía muy bien qué era lo que teníamos que hacer, todos comenzamos de cero" (E_ED_1), lo que es evidenciado en la actualidad, donde los distintos equipos de profesores trabajan desde diversas acciones a realizar, no existiendo un lineamiento específico de trabajo.

Además se identificó que no se evalúa formalmente el trabajo colaborativo, y este se desarrolla en base a la percepción y opiniones de los profesores, así se señala que: "no existe una evaluación formal, lo vamos viendo con el logro de los objetivos en los niños, en la medida que ellos van aprendiendo vemos que nuestro trabajo es fructífero" (E_PB_1), lo que coincide con: "un instrumento de evaluación para evaluar no hemos hecho, solamente la percepción de cada uno, de cómo ha dado resultado, si no ha dado resultado, en qué estamos fallando"(E_PB_2). Las respuestas señaladas coinciden con la observación, puesto que en éstas, los profesores no evaluaron el trabajo realizado, ni generaron planeaciones acerca de una futura evaluación como pares.

Otros factores que fueron identificados son el aumento de inasistencias por parte de los profesores. En las primeras semanas de observación se encontraban todos los profesores presentes, para finalmente asistir solo 8 docentes. El trabajo colaborativo de la escuela posee facilitadores principalmente orientados a la posibilidad de compartir en un horario protegido; pero además la existencia de obstaculizadores de múltiples índoles.

\section{Discusión}

A partir de los resultados de la investigación, se desprende que, considerando el primer objetivo, en la institución existe una organización para llevar a cabo el trabajo colaborativo que evidencia una secuencia de acciones respaldadas por el MINEDUC (2017), que dan cuenta de 
cómo se organiza el plan de estudio en conjunto (elección de un objetivo, estrategias diversificadas, estrategias de organización escolar, accesibilidad y participación de todos los que estudian la planificación y se debe abordar desde el DUA), en que las acciones desarrolladas son pertinentes, pues permite llevar a cabo aspectos claves del trabajo colaborativo.

En este trabajo colaborativo se fortalece la relación con la dupla, dado el proceso dialógico que ello implica. Esto coincide con lo planteado por Herrero (2012), quien señala que la interacción es un medio vinculado con la comunicación, en donde son los involucrados los que construyen su diálogo para interactuar. En este caso, se genera una comunicación directa entre los distintos profesores, lo que permite que ambos profesionales se complementen y se nutran de sus propias experiencias.

Rostein et al., (2006) señalan que todos los miembros aprecian el esfuerzo y aporte del otro, compartiendo una meta orientada al cumplimiento del "derecho a la educación con igualdad de oportunidades, permanencia y participación y logro de todos los estudiantes" (ver Juárez, Comboni y Garnique 2010, p. 49), lo que se ve claramente evidenciado en la escuela, puesto que los profesores destacan que trabajar en conjunto es un aporte, considerando que la comunicación genera un proceso de retroalimentación mutua de su quehacer educativo.

En relación al objetivo específico número 2, se han identificado ciertos facilitadores, tales como: el trabajo en un horario y lugar en común entre todos los profesores, lo cual es un factor innovador, ya que en la mayoría de las escuelas los profesores deben organizar y establecer un acuerdo en donde realizarán el trabajo colaborativo. Esto muchas veces no resulta beneficioso porque los tiempos no coinciden o es muy escaso. Estas evidencias son respaldadas por Rodríguez y Ossa (2014), quienes en su investigación evidenciaron que los profesores destacan que no poseen horas suficientes para realizar el trabajo colaborativo, por lo que deben “...utilizar tiempo informal por causa de problemas de contratación y coordinación de las horas entre los docentes" ( $\mathrm{p}$. 308). Esto claramente es un problema al que se dio solución en esta escuela, a través de la fijación de un horario y espacio en común para llevar a cabo el trabajo colaborativo, trayendo múltiples beneficios y 
facilidades para todos los profesores. De esta manera, Huguet (2009) menciona que los centros educativos deben dar "espacios de colaboración entre docentes para fomentar el apoyo mutuo y desarrollar relaciones de trabajo significativas" (p. 85).

A partir de los resultados de la investigación, se destaca como otro facilitador la voluntad de colaborar, puesto que los profesores ejercen el trabajo colaborativo, compartiendo experiencias de enseñanza-aprendizaje que permitan propiciar el aprendizaje de todos los estudiantes, adoptando de esta manera una actitud positiva hacia las necesidades de los estudiantes, eliminando así una de las barreras más constantes dentro de las escuelas. Tal como señala Huguet (2009), quien expresa que trabajar en colaboración "...favorece la valoración de las dificultades con las que se han encontrado los alumnos, la exploración de nuevas alternativas, la adopción de retos compartidos y la experimentación de nuevas posibilidades" (p. 86). Por este motivo, la nueva modalidad de trabajo colaborativo, que está ejerciendo la escuela investigada, permite la eliminación de barreras actitudinales por parte del profesorado.

Sin embargo, existen obstaculizadores que afectan el desarrollo del trabajo colaborativo, en donde se evidencia que los profesores no siempre cuentan con las planificaciones a la hora de desarrollar dicho trabajo y esto incide en las tareas a desarrollar durante el horario protegido. Son múltiples los autores que buscan explicar esta situación, sin embargo, Berrios et al., (2016) se focaliza en que en las instituciones educativas surgen problemáticas asociadas al liderazgo, puesto que el profesor regular ejerce un liderazgo por sobre el diferencial, lo cual incide en las distintas actividades que desarrollan en conjunto. Por este motivo constituye un desafío institucional que se sugiere sea abordado.

UNESCO (2000) atribuye que uno de los elementos necesarios para ejecutar un buen trabajo en equipo (colaborativo) es el liderazgo compartido; "Una responsabilidad compartida es la consideración de que, al poder tomar decisiones, todos pueden afectar la vida de los demás" (p. 16), es decir que, para resolver la dificultad, es necesario focalizarse en elementos de comunicación y responsabilidades compartidas que propicien el liderazgo. 
Otro obstaculizador presente es la falta de orientaciones de trabajo, donde los profesionales reconocen una inexistencia de los lineamientos o de orientaciones para desarrollar el trabajo colaborativo, repercutiendo en que cada uno realiza el trabajo como estime correcto. Ante esto, el MINEDUC (2015) señala que el PEI es un instrumento que contiene los distintos lineamientos institucionales, abordando el trabajo que se desarrolla para que la comunidad educativa tenga el conocimiento; también en el MINEDUC (2017), se señala que los lineamientos bases del trabajo colaborativo deben estar orientados desde el PME institucional. Si bien el PEI y PME no fueron estudiados en la presente investigación, existe la necesidad de potenciar los instrumentos para lograr un trabajo efectivo por toda la comunidad educativa.

Frente a la inexistencia de una evaluación formal del trabajo colaborativo, es importante señalar que MINEDUC (2015) establece una obligatoriedad de evaluación anual al considerar la gestión institucional y con ello las prácticas institucionales y pedagógicas. Sin embargo, al referirnos al trabajo colaborativo, Johnson, Johnson y Holubec (1999) plantean que uno de los elementos indispensables para un desarrollo oportuno es la autoevaluación grupal, por lo que se sugiere implementar una evaluación que permita reconocer el desarrollo personal y social, identificando los progresos y las falencias que aún deben ser mejoradas.

\section{Conclusiones}

A partir del análisis de la investigación realizada, se considera que, los cambios a nivel legislativo en Chile, han permitido que las instituciones educativas desarrollen un Trabajo Colaborativo, existiendo libertades en su organización. Ante esto, la nueva modalidad desarrollada en la escuela es innovadora y posee beneficios que fortalecen al ejercicio profesional de los distintos agentes de la educación, sin embargo, también están presentes las necesidades de mejora que permitan la constancia y desarrollo efectivo del trabajo colaborativo.

Para llevar a cabo esta modalidad de trabajo colaborativo es preciso continuar con el horario y lugar establecido, ya que esto posibilita que 
los profesionales se reúnan de manera certera, propiciando su desarrollo profesional en la escuela.

El proceso de este trabajo se organiza a través de acciones creadas por los profesores, lo cual es un aspecto positivo, sin embargo, es preciso que se planteen las orientaciones o lineamientos para desarrollarlo, ya que en la institución no se cuenta con ello y podría potenciar el trabajo realizado.

Otro de los elementos importantes es que los profesionales de la escuela tienen voluntad para colaborar, adoptando una actitud positiva hacia las necesidades de los estudiantes, porque es un factor que generalmente se ve debilitado en las instituciones educativas.

Para finalizar el proceso de trabajo colaborativo de manera efectiva, se requiere que se realicen procesos de evaluación constante sobre lo que se está llevando a cabo entre los profesores y a nivel institucional. Es necesario que los aspectos mencionados sean abordados en la institución educativa, sin embargo, también es indispensable que se brinde una solución, desde la formación inicial docente, en las diferentes casas de estudio de educación superior, para potenciar un aprendizaje sobre lo que significa o implica trabajar de forma colaborativa, en pro de una mejora para las futuras prácticas pedagógicas.

\subsection{Recomendaciones para la institución}

A partir de lo investigado, se recomienda a la escuela continuar con la nueva modalidad de trabajo colaborativo, creando un proceso de monitoreo para su continuidad, considerando los distintos facilitadores y obstaculizadores evidenciados. También, se recomienda evaluar el inicio, proceso y final del trabajo colaborativo para generar acciones de mejora.

\subsection{Recomendaciones para la formación inicial docente}

Se recomienda que, en el proceso de formación de los profesores, se entreguen conocimientos respecto a la modalidad de trabajo colaborativo entre profesores, permitiendo identificar y comprender el proceso a realizar y su importancia. 
Además, sería propicio generar instancias en que se lleve a cabo este tipo de trabajo para su dominio y conocimiento práctico.

\subsection{Limitaciones}

Las limitaciones que se presentaron fueron que, en un principio, se planeó entrevistar a todos los profesores de educación básica de la escuela que trabajaban en conjunto con los profesores de educación diferencial, pero finalmente, sólo se entrevistó a dos de ellos. Además, se planificó observar seis veces el desarrollo del trabajo colaborativo, pero por temas de tiempo académico, sólo se lograron observar cuatro de ellas.

Desde otra perspectiva, la investigación realizada desprendió información relevante que suscita la influencia de aspectos personales que influyen en el abordaje del trabajo colaborativo, como es, por ejemplo, la disposición a trabajar con pares. Es por ello que se debe mantener siempre una mirada objetiva para no generalizar las percepciones $y$ acciones de cada profesor.

\subsection{Proyecciones de la investigación}

Las nuevas investigaciones que pueden surgir a raíz de la presente dicen relación con el trabajo colaborativo en otras instituciones educativas o con otros profesionales. También sería interesante analizar su aplicabilidad en el aula, es decir, visibilizar el impacto del trabajo colaborativo por medio de la co-docencia.

\section{Agradecimientos}

Esta investigación fue posible gracias a la directora María Urenda y al ex jefe de la Unidad Técnica Pedagógica de la Escuela José Arbulú, quienes desde un principio nos abrieron las puertas y nos orientaron en nuestra investigación. Además, se les agradece a los profesores de educación diferencial y educación básica que decidieron participar en esta investigación educativa. 


\section{Referencias}

Abela, J. (2002). Las técnicas de Análisis de Contenido: Una revisión actualiza da. Recuperado de: http://www.albertomayol.cl/wp-content/uploads/ 2014/08/Andreu-Analisis-de-contenido.pdf

Anderson, P. (2002). Assesment and Devolopment of Executive Function (EF) During Childhood. Journal Child Neuropsychology, 8, 71-82.

Ardoy, L., Pasadas del Amo, S., y Ruíz, J. (2004). La triangulación metodológica en el ámbito de la investigación social: dos ejemplos de uso. Instituto de Estudios Sociales de Andalucía (IESA/CSIC). Departamento de Estudios telefónicos (España).

Berrios, N., Calderón, R., y Pizolty, R. (2016). Educación Inclusiva Escuela San Ignacio de Loyola de Valparaíso. Recuperado de: http://mailing.uahurtado.cl/cuadernos_educacion_75/documentos/Apoyo\%20al\%20docente_75.pdf

Canal, N.(2006). Técnicas de muestreo. Sesgos más frecuentes. Revista Seden, 13 (15), 121-132.

Cotrina, M., García, M., y Caparrós, E. (2017). Ser dos en el aula: Las parejas pedagógicas como estrategia de formación inicial del profesorado de secundaria. Aula Abierta, 46(0), 57-64.

Dávila, G. (2006). El razonamiento inductivo y deductivo dentro del proceso investigativo en ciencias experimentales y sociales. Revista de Educación Laurus, 12, 180-205.

Flick, U. (2004). Introducción a la investigación cualitativa. Madrid. Morata.

Font, J. (2009) La colaboración de los centros de educación especial a la inclusión. En Giné, C., Durán, D., Font, J. y Miquel, E. (Eds.), La educación inclusiva. De la exclusión a la plena participación de todo el alumnado. (pp. 81-94). Barcelona, Universitat de Barcelona, Institut de Ciències de l'Educació, ICE: Horsori.

Godoy, P., Meza L., y Salazar A. (2004). Antecedentes históricos, presente y futuro de la educación especial en Chile. Santiago de Chile: Ministerio de Educación.

Herrero, P. (2012). La interacción comunicativa en el proceso de enseñanzaaprendizaje. RelDoCrea, Revista electrónica de investigación Docencia Creativa, 1, 138- 143. 
Huguet, T. (2009). El trabajo colaborativo entre el profesorado como estrategia para la inclusión. En Giné, C., Durán, D., Font, J. y Miquel, E. (Eds.), La educación inclusiva. De la exclusión a la plena participación de todo el alumnado. (pp. 81-94). Barcelona, Universitat de Barcelona, Institut de Ciències de l'Educació, ICE: Horsori.

Jiménez, C. y Jiménez, E. (2004). Colaboración entre docentes para promover el aprendizaje del lenguaje en la primaria. Revista Iberoamericana de Educación. Recuperado de: https://rieoei.org/historico/deloslectores/593 Jimenez.pdf

Johnson, D; Johnson R, y Holubec, E (1999). Los componentes esenciales del aprendizaje cooperativo. En D.W. Johnson y R.T.y E. Holubec (Eds.) Los nuevos círculos del aprendizaje (pp. 37-41). Buenos Aires: Aique.

Juárez J., Comboni, S., y Garnique, F. (2010). De la educación especial a la educación inclusiva. Argumentos, 23(62), 41-83.

López, K. (2011). Factores que facilitan o dificultan el Trabajo Colaborativo en la Escuela Preparatoria Oficial $N^{\circ} 111$. Recuperado de: https://repositorio.tec.mx/bitstream/handle/11285/570584/DocsTec_11533. pdf? sequence $=1$ \&isAllowed $=y$

Maldonado, M. (2008). Aprendizaje Basado en Proyectos Colaborativos: Una experiencia en Educación Superior. Laurus, 14(28), p. 158-180.

Merchán, C.y Ramírez,J. (2015). La observación de prácticas de aula: inicios de la práctica pedagógica efectiva. Dialnet, 14, 98-107.

MINEDUC (2005). Politica Nacional de Educación Especial: Nuestro Compromiso con la Diversidad. Recuperado de: http://especial.mineduc.cl/wpcontent/uploads/sites/31/2016/08/POLiTICAEDUCESP.pdf

MINEDUC (2008). Marco para la Buena Enseñanza. Recuperado de: http:// www.cpeip.c1/wp-content/uploads/2017/10/MBE.pdf

MINEDUC (2015). Decreto 83: Aprueba criterios y orientaciones de adecuación curricular para estudiantes con necesidades educativas especiales de educación parvularia y educación básica. Recuperado de: http://especial.mineduc.cl/ wp-content/uploads/sites/31/2016/08/Decreto-83-2015.pdf

MINEDUC (2017). Orientaciones sobre estrategias diversificadas de enseñanza para educación básica, en el Marco del Decreto 83/2015. Recuperado de: http://especial.mineduc.cl/wp-content/uploads/sites/31/2017/05/ ORIENTACIONES_D83_Web_05-2017.pdf

Navarro, B., Arriagada, I., Osse, S., y Burgos, C. (2016). Adaptaciones 
curriculares: Convergencias y divergencias de su implementación en el profesorado chileno. Revista Electrónica Educare, 20, 1-18.

Quecedo, R. y Castaño, C. (2002). Introducción a la metodología de investigación cualitativa. Revista de Psicodidáctica, 14, 5-39.

Rodríguez, F. (2012). La percepción del trabajo colaborativo en la gestión curricular de profesores de educación regular y educación especial en Programas de Integración Escolar de la comuna de Tomé. Tesis de magíster no publicada, Universidad del Bío-Bío, Chillán, Chile.

Rodríguez, F. (2014). La co-enseñanza, una estrategia para el mejoramiento educativo y la inclusión. Revista Latinoamericana de Educación Inclusiva, 8,219-233.

Rodríguez, F. y Ossa, C. (2014). Valoración del trabajo colaborativo entre profesores de escuelas básicas de Tomé, Chile. Estudios pedagógicos, 40(2), 303-319.

Rotstein, B., Scassa, A.M., Sáinz, C. y Simesen, A.M. (2006). El trabajo colaborativo en entornos virtuales de aprendizaje. Revista Cognición, 7, p. 38-45.

Sanhueza, S., Granada, M., y Bravo, L. (2013). Actitudes del profesorado de Chile y Costa Rica hacia la inclusión educativa. Cadernos de Pesquisa, 42(147), 884-899.

UNESCO (2000). Competencias para la profesionalización de la gestión educativa. Recuperado de: https://www.buenosaires.iiep.unesco.org/publicaciones/competencias-para-la-profesionalizacio-n-de-la-gesti-n-educativa. 